\title{
Editorial
}

\section{Molecular Modeling: Advancements and Applications}

\author{
André Silva Pimentel, ${ }^{1}$ Cristiano R. W. Guimarães, ${ }^{2}$ and Yifat Miller ${ }^{3}$ \\ ${ }^{1}$ Departamento de Química, Pontifícia Universidade Católica do Rio de Janeiro, 22451-900 Rio de Janeiro, RJ, Brazil \\ ${ }^{2}$ Pfizer Worldwide Research \& Development, Cambridge, MA 02139, USA \\ ${ }^{3}$ Department of Chemistry, Ben-Gurion University of the Negev, 84105 Be'er-Sheva, Israel
}

Correspondence should be addressed to André Silva Pimentel; a_pimentel@puc-rio.br

Received 12 May 2013; Accepted 12 May 2013

Copyright (C) 2013 André Silva Pimentel et al. This is an open access article distributed under the Creative Commons Attribution License, which permits unrestricted use, distribution, and reproduction in any medium, provided the original work is properly cited.

Molecular modeling (MM) is one of the fastest growing fields in science. It may vary from building and visualizing simple molecules in three dimensions (3D) to performing complex computer simulations on large proteins and nanostructures. $\mathrm{MM}$ is a collection of computer-based techniques for driving, representing and manipulating the structures and reactions of molecules, and those properties that are dependent on these 3D structures. The techniques in MM cover several issues among them computational chemistry, drug design, computational biology, nanostructures, and material science.

This issue compiles eight papers that deal with five issues: new techniques in MM, computational chemistry, computational biology, nanostructures, and material science.

Two new techniques are described in this issue. The first technique is presented by D. J. Medeiros et al. who developed a utility program to compute ab initio charges of each atom for relatively large molecules. The second technique is presented by K. Dedachi et al. that developed a new all-atom force-field in applications for 3D structural prediction for short peptides.

Two papers that are related to computational chemistry in this issue are by S. Arshadi et al. One paper illustrates the applications of density functional theory (DFT) to investigate the properties of the electronic structure of Zigzag boron nitride nanotubes and two models of diborinin-doped boron nitride nanotube. The second paper presents the applications of DFT to investigate the electronic structure of pyrazinedoped nitride nanotubes and to compute the nuclear magnetic resonance (NMR) parameters of these structures.

One of the new fields in MM is the study of the selfassembly of molecules that forms nanostructures. The paper by R. Behjatmanesh-Ardakani and M. Farsad illustrate a MM of critical micelle concentration for a single chain and a double chain of a surfactant with amphiphilic properties. The self-assembly processes of the single chain and the double chain surfactants are compared using Monte-Carlo simulations.

Two papers deals with computational biology in aim to understand biological mechanisms. One paper by X. Liu et al. demonstrates the interaction mechanism of transresveratrol with bovine serum albumin using a combination of MM techniques and spectroscopic methods. The second paper by I. O. Akdag and E. Ozkirimli demonstrates the applications of steered molecular dynamics simulations to examine the membrane translocation mechanism of the $\mathrm{p}$ VEC peptide. This short peptide is derived from murine vascular endothelial-cadherin protein that functions in the physical contact between adjacent cells.

Finally, one of the main advancements in the last five years is the study of material science using MM. The paper by T. Guerra and I. Borges Jr. illustrates this advancement using a combination of DFT with periodic boundary conditions and generalized gradient approximation methods. The authors succeeded to describe the electronic charges density of the adsorbed 2,4,6-trinitrotoluene on a $\mathrm{MgO}$ (001) surface in aim to understand the interactions between the molecules and the surface. This issue does not include peer-reviewed articles on drug design. Yet, we found that the issue drug design is an interactive topic in MM and contribute to drug discovery both in academia and in industry. Computer-aided and structure-based drug design relies on knowledge of the 
3D structure of the biological target. Drug design is an iterative process that begins when a compound is identified to display an interesting biological profile and ends when its activity profile and the chemical synthesis are optimized. Today, MM permeates all aspects of drug design. Scientists have used computer models of new chemical entities to help define activity profiles, geometries, and relativities. Three stages of drug discovery can be achieved by MM: virtual screening, hit-to-lead optimization of affinity and selectivity, and lead optimization of other pharmaceutical properties while maintaining affinity.

We thoroughly reviewed these papers and we believe that they contribute to the advancements and the applications of new MM techniques for both chemical and biological molecules. Moreover, we believe that the papers in this special issue will be milestones of the advancement for the next generation of MM researchers.

Yifat Miller

Cristiano R. W. Guimarães André S. Pimentel 

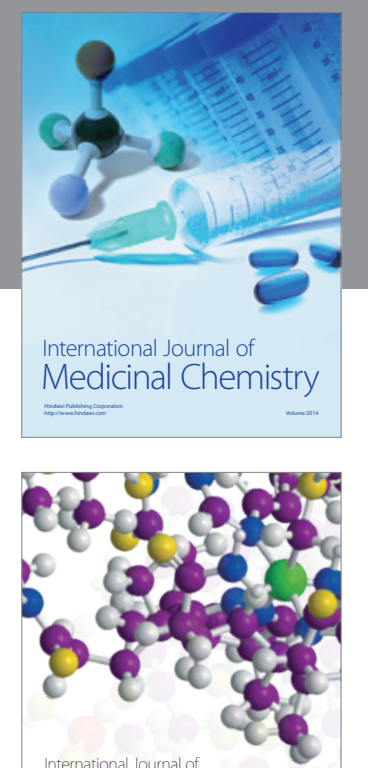

\section{Carbohydrate} Chemistry

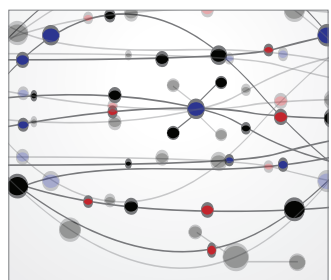

The Scientific World Journal
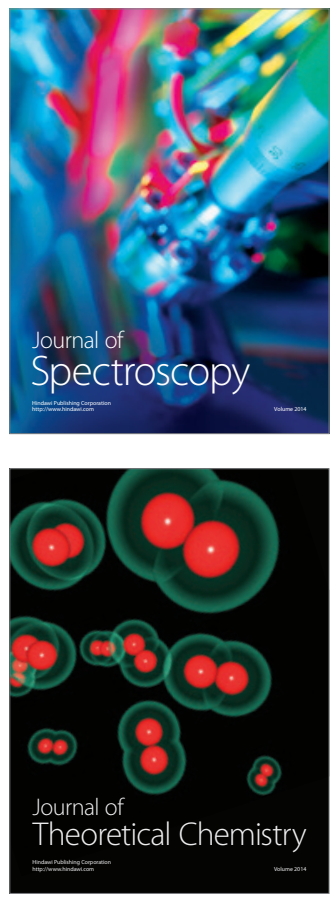
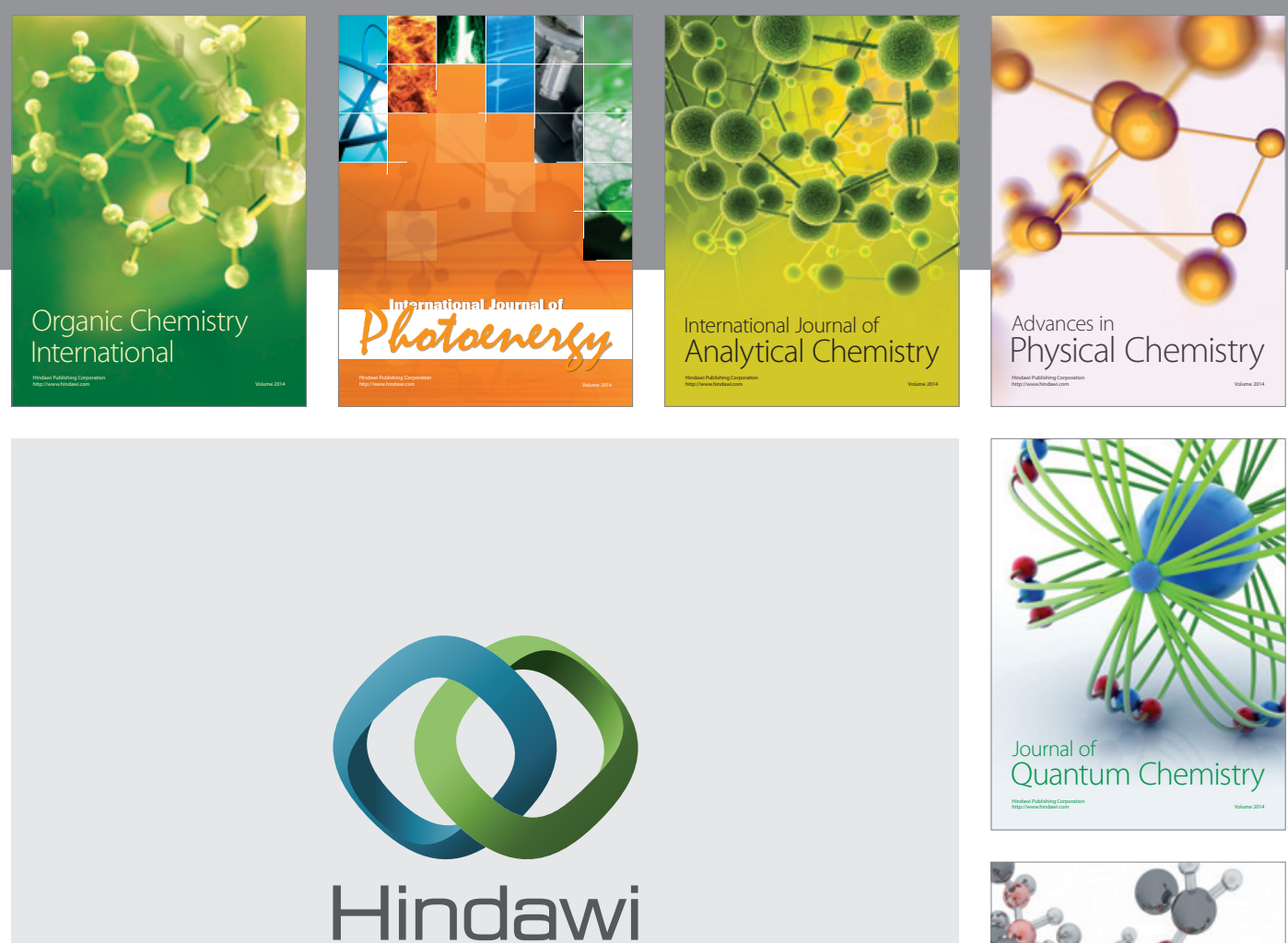

Submit your manuscripts at

http://www.hindawi.com

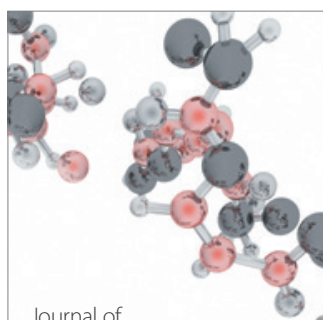

Analytical Methods

in Chemistry

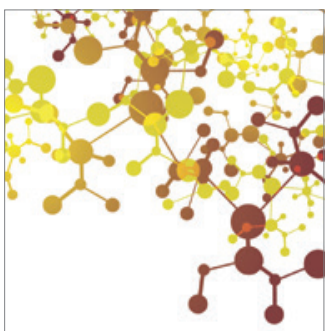

Journal of

Applied Chemistry

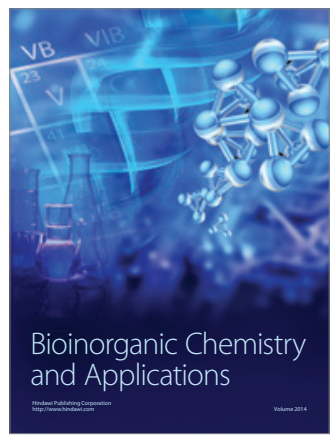

Inorganic Chemistry
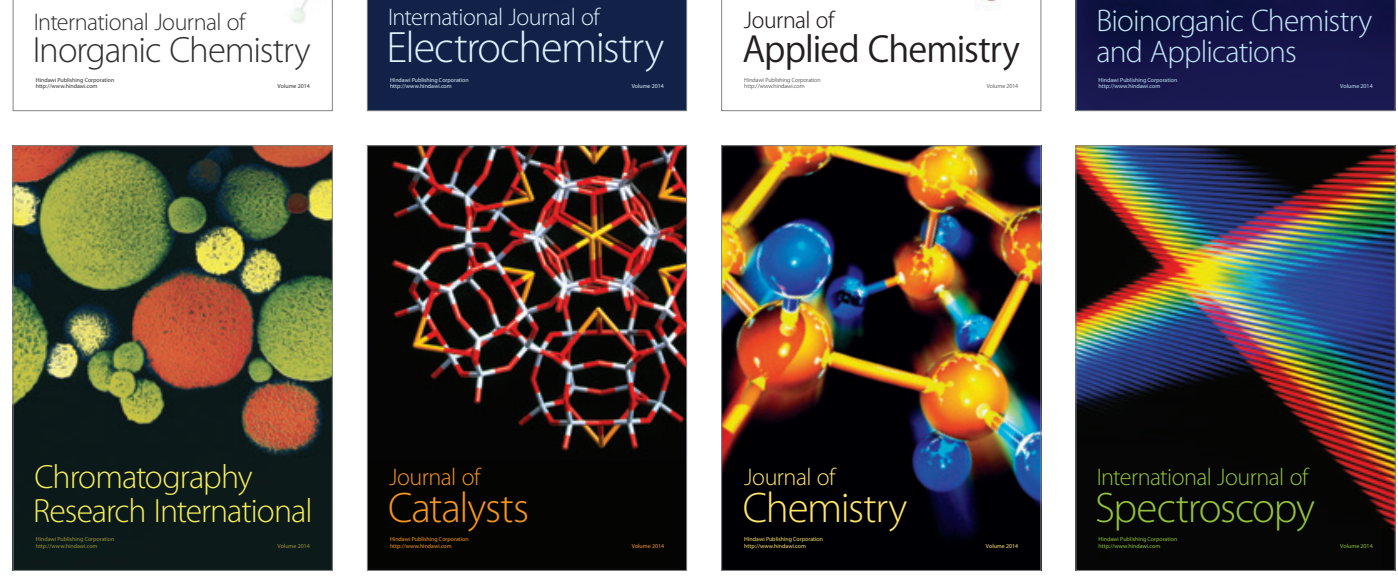